\title{
The common use of improper control diets in diet-induced metabolic disease research confounds data interpretation: the fiber factor
}

\author{
Michael A. Pellizzon* and Matthew R. Ricci
}

\begin{abstract}
Diets used to induce metabolic disease are generally high in fat and refined carbohydrates and importantly, are usually made with refined, purified ingredients. However, researchers will often use a low fat grain-based (GB) diet containing unrefined ingredients as the control diet. Such a comparison between two completely different diet types makes it impossible to draw conclusions regarding the phenotypic differences driven by diet. While many compositional differences can account for this, one major difference that could have the greatest impact between GB and purified diets is the fiber content, both in terms of the level and composition. We will review recent data showing how fiber differences between GB diets and purified diets can significantly influence gut health and microbiota, which itself can affect metabolic disease development. Researchers need to consider the control diet carefully in order to make the best use of precious experimental resources.
\end{abstract}

Keywords: Diet, Purified ingredients, Fiber, Microbiota, Metabolic disease

\section{Perspective}

Animal models have been and continue to be crucial in understanding the etiology of metabolic disease in humans. One reason is that similar to humans, metabolic disease in lab animals can be induced by diet. Unlike human clinical work, researchers have the opportunity to carefully and easily control the animal's environment which should include the diet. Unfortunately, there are a substantial number of studies in which improperly matched experimental and control diets are used. Such mismatched diets hamper the investigator's ability to draw useful conclusions from what are otherwise well-designed, hypothesis-driven studies. One recurring example is the comparison of a defined, purified ingredient diet to something often referred to as 'normal chow' or 'standard laboratory chow', which typically refers to a grain-based (GB) diet (see Table 1). The term 'normal chow' is about as useful a description for a diet as 'normal mouse' would be for an animal. In addition, 'normal chow' suggests that 1) all chows have a consistent and known composition

\footnotetext{
* Correspondence: michael.pellizzon@researchdiets.com

Research Diets, Inc, 20 Jules Lane, New Brunswick, NJ 08901, USA
}

and 2) the use of chow as the control diet is always acceptable, neither of which is true.

In this essay, our goal is to educate researchers that lab animal diets must be properly controlled and reported so that valid conclusions can be made. The use of improper control diets in metabolic disease research and the lack of adequate diet descriptions in publications has been discussed previously [1-6]. However, revisiting this topic is crucial and timely given the 1) substantial and widespread interest in the role of the gut microbiome in health and disease and 2) the undisputed effects of diet on gut microbe activity and populations. In order to appreciate why comparing these different diet types is not good science, let's first define these diets.

\section{Complexity of GB diet ingredients can confound data interpretation}

GB diets, which are often and unfortunately referred to vaguely as "normal chow", "normal diet" or "standard diet" in most publications, are made with grain and cereal ingredients and animal by-products. These unrefined ingredients include 'ground corn,' 'ground wheat,' 'ground oats', 'fish meal', 'alfalfa meal', 'brewers dried yeast' and 
Table 1 Typical sources of nutrients and non-nutrients in rodent purified ingredient diets and grain-based diets

\begin{tabular}{lll}
\hline $\begin{array}{l}\text { Nutrients or } \\
\text { Non-nutrients }\end{array}$ & Purified Ingredient Diet & Grain-Based Diet \\
\hline \multirow{2}{*}{ Protein } & Typical Sources & Typical Sources \\
Fat & Casein & Dehulled soybean meal, ground corn and wheat, whey, alfalfa \\
Carbohydrate & Soybean oil, corn oil & Porcine animal fat, fish meal, meat meal \\
Fiber & Refined Cellulose (INSOLUBLE Fiber) & Ground corn or wheat, dried beet pulp, ground oats, alfalfa, wheat middlings \\
& & (SOLUBLE and INSOLUBLE Fibers including cellulose, hemicellulose, lignins and pectin) \\
Micronutrients & Vitamin and mineral premixes & Most ingredients, extra micronutrients added \\
Phytoestrogens & None present in diet & Mainly soybean meal, alfalfa meal \\
Heavy Metals & None present in diet & Mainly from grains and meat meals \\
\hline
\end{tabular}

'animal fat preserved with BHA'. These ingredients contain multiple nutrients and non-nutrients, and their inclusion level in GB diets (i.e. the formula) is not only 'closed' (kept secret from the research community) as it is considered proprietary, but the formula itself may vary over time depending on changes in nutrient levels in key ingredients. In addition, vitamin and mineral premixes are added to these diets which supplement the unknown levels of micronutrients provided inherently from other ingredients, and in some cases in excess of the estimated requirement [7]. One the positive side, GB diets are inexpensive and have been used since the beginning of lab animal research. In addition, they are generally considered to maintain a healthy phenotype in the animal (though it can be argued 'compared to what?').

However, the ingredients used in GB diets are also their Achilles heel. The extent to which these ingredients are processed and the locations and conditions of where they are harvested can be a source of variation in the nutrients and non-nutrients (such as phytoestrogen levels) they contain $[8-10]$. Therefore, GB diets can vary significantly from batch-to-batch, from formulation to formulation and among different manufacturers. While variation in nutrient content alone should make a researcher reconsider the use of a GB diet, the presence and inconstancy of non-nutrients (which are generally not listed on the nutrition label) in these diets further adds to these concerns. Conceivably, this may inadvertently change the research question being asked in the study, leading to more time and money spent.

There is a growing list of non-nutrient entities in GB diets such as various phytochemicals (e.g. phytoestrogens, lignans) [11], toxic heavy metals (e.g. arsenic, lead) [12], nitrosamines [13, 14], endotoxins [15] and pesticides and pollutants [16-18]. Recently, Mesnage et al. [18] looked at 13 different GB diets from 5 continents and found several environmental contaminants including various pesticides, heavy metals, genetically modified grains, polychlorinated biphenyls, polychlorinated dibenzo-p-dioxins and dibenzofurans. Their levels in some cases greatly exceeded acceptable daily intakes and are highly variable among these diets. As it is apparent that these contaminants are not well controlled, it's conceivable that they could by themselves or in combination alter the toxicological and metabolic phenotype of rodents. For example, it was found that feeding a GB diet significantly induced expression of aryl hydrocarbon receptors (AhRs) in intestinal cells - cells that modulate immunity and detoxification [19]. In contrast, expression was not induced by feeding a purified ingredient diet. However, the addition of a known AhR ligand (indole-3 carbinol) to a purified diet recapitulated the effect of the GB diet. While indole-3 carbinol is not present in GB diets, it is likely that other phytochemicals (i.e. phytoestrogens from soybean meal and alfalfa meal) or perhaps environmental contaminants such as polychlorinated dibenzo-p-dioxins may serve as AhR ligands [20].

In contrast to GB diets, purified ingredient diets (also called purified diets, semi-purified diets) use highly refined ingredients (casein, corn starch, sucrose, cellulose, soybean oil, etc.) each of which essentially contains one main nutrient and little to no non-nutrient chemicals. As a result, these diets are well-defined and have minimal batch-to-batch variability [21]. Indeed, it was through the use of purified ingredient diets that nutrient requirements for lab animals were first delineated. Furthermore, the formulas are 'open' and not kept secret from the scientific community. No diet is without flaws and a good example of a needed 'improvement' in purified diets is the inclusion of a source(s) of soluble fiber for gut health. Having said this, among nutritionally trained scientists, purified diets are considered a 'cleaner', more controlled diet choice compared to GB diets $[4,7,21]$. Knowing the inherent differences between GB diets and purified diets allows the researcher to design their diet study well and judge papers in which animals fed purified diets were compared directly to those fed GB diets.

Given the inherent differences between GB and purified diets, it is clear that data produced from these diets should not be compared to each other. Yet, the incidence of improperly controlled diet studies in the lab animal literature 
is substantial. In 2008, Warden and Fisler [5] identified 35 papers using the search terms 'mouse high fat' in five high-impact journals. Of these 35 papers, only $14 \%$ used a properly matched control diet against the high-fat purified ingredient experimental diet. Forty-three percent of papers improperly used a GB diet as the control and 34\% of the time, there was not enough information in the methods section to even determine what types of diets were fed. Despite this high-profile commentary, these numbers have not changed very much in recent years. We used the same search terms in the same journals, and identified 69 publications published in 2016. In $41 \%$ of the papers, data from mice fed a high-fat purified ingredient diet were improperly compared to those fed a GB diet. In another $41 \%$ of the papers, there were insufficient descriptions of the diets used and so we could not determine what the animals were fed. In only $19 \%$ of the papers was a properly matched, low-fat purified ingredient diet used in comparison to the purified ingredient high-fat diet (Fig. 1). Thus, it is possible that researchers are attributing phenotypic differences between animals fed a low fat GB diet and a high fat purified diet to differences in dietary fat, when in fact they could be due to any number of other dietary differences.

Interestingly, the authors of a recent publication [22] compared feeding a low-fat GB diet, a high-fat purified

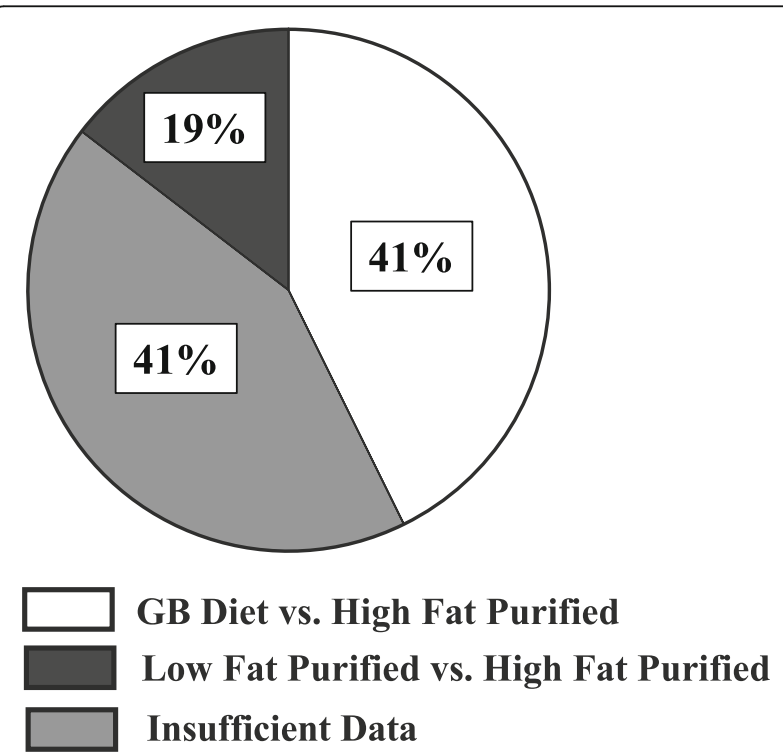

Fig. 1 Diet comparisons in recent research publications. Pie chart showing the percentages of 69 publications evaluated (using search terms 'mouse high fat') that used appropriate diet comparisons (19\%), that compared GB diets and purified high-fat diets (41\%), and that presented insufficient information to evaluate the types of diets used (41\%). The journals examined were Cell Metabolism (7 papers), Cell (1 paper), Science (1 paper), Journal of Clinical Investigation (15 papers), Nature (3 papers), Nature Medicine (4 papers), and Diabetes (the first 38 of 188 papers) diet and a matched low-fat purified diet to $\mathrm{C} 57 \mathrm{Bl} / 6 \mathrm{~J}$ mice. As they found no differences in body weight, glucose tolerance, adipokines or anxiety-like behavior between the GB or low-fat purified diet, they concluded that "chow (GB diet) may be used as an appropriate control diet in studies investigating the effects of chronic high-fat diet intake on phenotypic, metabolic and behavioral alterations". In our opinion, this is an overreaching and misleading statement to make, given that their study, like any other, was limited in the scope of the endpoints measured. In their study, they did find differences in plasma lipids levels between the GB and low-fat purified diets, something they acknowledge could be due to differences in dietary fiber between these diet types. However, the authors fail to note that dietary fiber differences may (and based on data from the literature, will) affect gut morphology and the microbiome, an area of intense research. This highlights a relevant but overlooked confound that occurs any time a high fat purified diet is compared to a GB diet: the vastly different fiber types and concentrations between these diet types.

There is a growing body of evidence that the relationship between diet and metabolic disease is gut-centric. The link between the gut and weight gain was established by Turnbaugh et al. [23] who observed that lean, germfree mice gained more adiposity after they were gavaged with cecal microbiota from obese $o b / o b$ mice compared to those gavaged with microbiota from lean mice. Dramatic shifts in certain bacterial phyla can occur within a day after switching from a 'lower fat, plant polysaccharide based diet' (an undefined GB diet) to a high fat purified ingredient diet, and this stabilizes after only 7 days [24]. These intriguing findings suggest a rapid and powerful effect of diet on changing microbiota which precedes the development of metabolic disease. While these studies were significant contributions to our understanding of the role of gut microbiota on metabolic disease, the fact that the descriptions of the diets were limited reduces our understanding of which particular dietary factor(s) were important to the observations.

\section{Fiber: An X factor in GB diets that has been ignored}

Of the many differences between GB and purified ingredient diets, it is arguably the level and type of dietary fiber which is most important with respect to the gut microbiome. Fiber can be generally classified as either soluble or insoluble, and there are different types of each. Bacterial fermentation of soluble fiber releases short chain fatty acids (SCFAs), which are a major supplier of energy to colonocytes and are thought to provide other benefits including prevention of diet-induced obesity, decreased adipose tissue storage, and improved insulin action [25]. An increase in SCFAs can change the gut $\mathrm{pH}$, which in turn can decrease the populations of pathogenic, $\mathrm{pH}$ - 
sensitive bacteria. In contrast, insoluble fiber is generally considered to be non-fermentable and therefore does not promote gut bacterial growth and its associated effects [26]. Given these important differences in fiber, researchers working in the gut microbiota field need to educate themselves regarding the fiber level/type in the diets being fed to their animals.

What then, are the specific differences in fiber content between GB and purified ingredient diets? Crude fiber in commercial GB diets is typically reported at $5-6 \%$, but in reality, the total fiber level in commercial GB diets varies much more and total levels are around 15-25\% ( 15-20\% insoluble and 2-5\% soluble, unpublished data, Pellizzon and Ricci). Fiber sources in GB diets include a mixture of cellulose, hemicellulose, lignins, and pectin, and their levels can vary significantly among different chows [27]. This is not surprising given the variable levels of fiber-containing, grain-based ingredients added to different GB diets, including ground corn, wheat, and oats, wheat middlings, soybean and alfalfa meal, dried beet pulp, and brewers dried yeast. In addition, each of these ingredients may vary in fiber level. For example, wheat middlings, a byproduct of wheat milling, can vary in fiber content (as well as other nutrients), depending on their harvest location [28]. Because of the above mentioned complexities of GB diet ingredients and their changing inclusion levels, it is impossible to know the total fiber levels and concentrations of each fiber type from diet-to-diet or even from batchto-batch. In contrast, purified diets in general (including high- and low-fat diets) have historically contained 5\% fiber, typically with refined cellulose (an insoluble fiber) as the sole fiber source. Arguments can be made that it is time to rethink the fiber component in purified diets, since it is likely that $5 \%$ cellulose is not a sufficient amount or type of fiber for optimal gut health.

In addition to the multiple differences between GB diets and purified ingredient diets already discussed, the disparity in fiber level and type alone would be expected to have important experimental consequences given their wellknown effects in the intestine including adsorption of bile acids, chelation of minerals, and fermentation by bacteria in the cecum and colon. It has been known for decades that colon and cecum morphology is affected differently by GB diets and purified diets, an effect that is attributed to the differences in fiber composition of the diets [29]. Thus from a fiber perspective (in addition to others already discussed), animals fed purified ingredient diets should never be compared to those fed a GB diet. This is highlighted in a recent study by Chassaing et al. [30] and demonstrates the erroneous conclusions that can be drawn from mismatched diets. These researchers found that the colons and ceca of animals fed a high-fat purified ingredient diet were significantly smaller than those fed a low-fat GB diet. Had the GB diet been their only 'control', it might have been tempting to conclude that the changes in gut morphology were due to differences in dietary fat. However, when the mice were fed a low-fat purified diet (matched in every way to the high-fat purified diet, except in fat and carbohydrate) the mice had gut morphologies identical to those fed the high-fat diet. As there are a number of differences between these different types of diets that may have a potential influence on gut morphology, these investigators selected 2 very different factors in these diets, which were the protein type (casein vs. soy) and fiber contents. Specifically, these investigators examined if changes in protein type (casein vs. soy protein, which provided phytoestrogens), fiber levels (50 g, $100 \mathrm{~g}$, and $200 \mathrm{~g}$ per $\sim 4000 \mathrm{kcals}$ ), and fiber types (insoluble fiber cellulose vs. soluble fiber inulin) influenced gut morphology. Through a number of experiments, the authors showed that it was most likely the differences in dietary soluble fiber type (and not the fat level or protein type) between the GB and the purified ingredient diets that were responsible for the differences in gut size. Very recently, Dalby et al. [31] extended some of the findings of this work showing that low and high fat purified diets (10 or $60 \mathrm{kcal} \%$ fat and matched with the same sucrose levels and fiber as only cellulose) altered small and large intestinal microbiota composition similarly and both were very different from GB diet fed mice. While both GB diet and purified low fat fed mice had similar but significantly lower body weight, adiposity, and glucose intolerance compared to high fat fed mice, the similarities in the microbiota composition (in ileum, cecum, and colon) and cecal SCFAs of purified low and high fat fed mice suggest that other changes were responsible for the effects on the rodent metabolic phenotype. The conclusions in these studies would have been impossible to make had the GB diet been the only 'control'. In another recent study, Desai et al. found that gnotobiotic mice with a synthetic human gut microbiota fed a purified low fat diet with very low fiber as cellulose had a significantly thinner colonic mucus layer compared to those fed a fiber-rich GB diet, something which was found to be driven by shifts in mucus-eroding microbiota and was associated with low grade inflammation and increased susceptibility to bacterial infection [32]. The differential effect of diet type on gut morphology and disease severity is also extended to mouse models of experimentally induced colitis. Miles et al. [33] observed that relative to a GB diet, feeding purified diets with either 10 or $60 \mathrm{kcal} \%$ fat with fiber as cellulose only increased weight loss and reduced cecum size and colon length. Again, had the GB diet been used as the only control diet, it may have been concluded that the fat level rather than diet type increased disease susceptibility. The above data suggest, not surprisingly, that changes in gut morphology and microbiota induced by diet are accompanied by changes in gut function and disease susceptibility. 
Based on the above data, the differences in fiber type between a GB diet and a purified diet would be expected to influence gut morphology and metabolic health. However the extreme differences in ingredient types between GB and purified diets suggest there may be additional dietary factors influencing health. While a shift from casein to soy protein in a purified diet may not significantly influence gut morphology to the same extent as changes in dietary fiber [30], there is evidence that it can affect metabolic disease development, including body weight gain, adiposity, and plasma and liver lipids [34]. In terms of gut health, many xenobiotic compounds (including flavonoids, arsenic, and polychlorinated dibenzo-pdioxins) in GB diets either by themselves or in combination could potentially mediate beneficial and/or toxic effects through binding gut $\mathrm{AhR}$ and subsequent modification of gut microbiota composition [20]. Given the complex and variable nature of GB diets as mentioned previously, it is difficult to determine the true influence of these factors on the rodent phenotype unless they are studied one at a time using a purified ingredient diet, as suggested previously [12]. Therefore, there are many potential factors in GB diets besides fiber type that can alter the rodent gut and metabolic phenotype from health to disease.

Many publications have shown the beneficial effects of adding soluble fiber to purified diets to improve health using rodent models. For example, in the context of purified ingredient diets, the addition of purified soluble fiber sources including inulin, fructooligosaccharides, and pectin can improve gut morphology [35-38] and reduce body weight and adiposity relative to insoluble cellulose $[30,38,39]$ in rats and mice. In addition, other fibers including those classified as hemicellulose (which are in GB diets) such as xylans, glucans, and mannans, can improve gut morphology [40] and reduce adiposity, inflammation, and improve glucose tolerance, all of which was associated with improved mucosal barrier function [41]. Therefore, should one wish to formulate a base purified control diet that would allow rodents to have an improved gut and metabolic health profile, one easy strategy to accomplish this is to use purified soluble fiber sources. This would allow the researcher to have a better ability to control for phenotypical differences within a given study and from study to study, which would not be possible when using a GB diet, which contain a complex (and likely variable) array of fibers.

Proper experimental design needs to include an understanding of the dietary components and their potential effects on the interpretability of the data. The diet is not 'just the food' but a key environmental factor that can and will affect the phenotype of the animals. Given the NIH's recent mandate to "enhance reproducibility of research findings through increased scientific rigor and transparency" in grant applications [42], researchers should be making efforts to optimize study design with respect to the diets fed. This will make the best use of funding resources, allow us to draw valid conclusions from these data and enhance our collective knowledge of these disease models. In the end, these endeavors will help us all reach our goal: the treatment and prevention of human metabolic disease.

\section{Abbreviations \\ AhR: Aryl hydrocarbon receptors; GB: Grain-based; SCFAs: Short chain fatty acids}

\section{Acknowledgements \\ Not applicable.}

Funding

Not applicable.

\section{Availability of data and materials}

Data sharing not applicable to this article as no datasets were generated or analysed during the current study.

\section{Authors' contributions}

MAP drafted the manuscript and MRR contributed to revisions and edits. Both MAP and MRR read and approved the final manuscript.

Ethics approval and consent to participate

Not applicable.

Consent for publication

Not applicable.

\section{Competing interests}

MAP and MRR are employees of Research Diets, Inc.

Received: 15 November 2017 Accepted: 10 January 2018

Published online: 15 January 2018

\section{References}

1. Fodde R, Schmitt M, Schewe M, Augenlicht LH. Fodde Hepatobiliary surgery 2017 commentary Modelling western dietary habits in the mouse. Hepato Biliary Surg Nutr. 2017;6(2):138-40.

2. Institute for Laboratory Animal Research. In: National Research Council, editor. Guidance for the description of animal research in scientific publications guidance for the description of animal research in scientific publications. Washington, D.C.: The National Academies Press; 2011.

3. Omary MB, Cohen DE, El-Omar EM, Jalan R, Low MJ, Nathanson MH, et al. Not all mice are the same: standardization of animal research data presentation. Gastroenterology. 2016;150(7):1503-4.

4. Rendina-Ruedy E, Smith BJ. Methodological considerations when studying the skeletal response to glucose intolerance using the diet-induced obesity model. Bonekey Rep. 2016;5:845.

5. Warden $\mathrm{CH}$, Fisler JS. Comparisons of diets used in animal models of highfat feeding. Cell Metab. 2008;7(4):277.

6. Kilkenny C, Browne WJ, Cuthill IC, Emerson M, Altman DG. Improving bioscience research reporting: the ARRIVE guidelines for reporting animal research. PLoS Biol. 2010;8(6):6-10.

7. General considerations for feeding and diet formulation. In: Subcommittee on laboratory animal nutrition, committee on animal nutrition, board on agriculture, National Research Council nutrient requirements of laboratory animals, fourth revised edition. Washington, D.C: National Academy Press; 1995. p. 3-10.

8. Greenman D, Oller W, Littlefield N, Nelson C. Commercial laboratory animal diets: toxicant and nutrient variability. J Toxicol Environ Health. 1980;6:235-46.

9. Newberne PM. Influence on pharmacological experiments of chemicals and other factors in diets of laboratory animals. Food Sources Incidental Drug Expo. 1975;34:209-18.

10. Mead MN. The feed factor: estrogenic variability in lab animal diets. Environ Health Perspect. 2006;114(11):A640-2. 
11. Jensen MN, Ritskes-Hoitinga M. How isoflavone levels in common rodent diets can interfere with the value of animal models and with experimental results. Lab Anim. 2007;41(1):1-18.

12. Kozul CD, Nomikos Athena P, Hampton TH, Warnke LA, Gosse JA, Davey JC, et al. Laboratory diet profoundly alters gene expression and confounds genomic analysis in mouse liver and lung. Chem Biol Interact. 2008:173(2):129-40.

13. Rao GN, Knapka JJ. Contaminant and nutrient concentrations of natural ingredient rat and mouse diet used in chemical toxicology studies. Fundam Appl Toxicol. 1987;9(2):329-38.

14. Silverman J, Adams JD. N-nitrosamines in laboratory animal feed and bedding. Lab Anim Sci. 1983;33(2):161-4.

15. Hrncir T, Stepankova R, Kozakova H, Hudcovic T, Tlaskalova-Hogenova H. Gut microbiota and lipopolysaccharide content of the diet influence development of regulatory T cells: studies in germ-free mice. BMC Immunol. 2008. https://doi.org/10.1186/1471-2172-9-65.

16. Coleman WE, Tardiff RG. Contaminant levels in animal feeds used for toxicity studies. Arch Env Contam Toxicol. 1979;8(6):693-702.

17. Schecter AJ, Olson J, Papke O. Exposure of laboratory animals to polychlorinated dibenzodioxins and polychlorinated dibenzofurans from commerical rodent chow. Chemosphere. 1996;32(3):501-8.

18. Mesnage R, Defarge N, Rocque LM, De Vendômois JS, Séralini GE. Laboratory rodent diets contain toxic levels of environmental contaminants: implications for regulatory tests. PLoS One. 2015;10(7):e0128429. https://doi. org/10.1371/journal.pone.0128429.

19. Ito S, Chen C, Satoh J, Yim S, Gonzalez FJ. Dietary phytochemicals regulate whole-body CYP1A1 expression through an arylhydrocarbon receptor nuclear translocator-dependent system in gut. J Clin Invest. 2007;117(7):1940-50.

20. Zhang L, Nichols RG, Patterson AD. The aryl hydrocarbon receptor as a moderator of host-microbiota communication. Current Opinion in Toxicology. 2017;2:30-5.

21. Wise A. Interaction of diet and toxicity-the future role of purified diet in toxicological research. Arch Toxicol. 1982;50:287-99.

22. Almeida-Suhett CP, Scott JM, Graham A, Chen Y, Deuster PA. AlmeidaSuhett nutritional neuroscience 2017 control diet in a high-fat diet study in mice: regular chow and purified low-fat diet have similar effects on phenotypic, metabolic, and behavioral outcomes. Nutr Neurosci. 2017:1-11.

23. Turnbaugh PJ, Ley RE, Mahowald MA, Magrini V, Mardis ER, Gordon JI. An obesity-associated gut microbiome with increased capacity for energy harvest. Nature. 2006;444(7122):1027-31.

24. Turnbaugh PJ, Ridaura VK, Faith JJ, Rey FE, Knight R, Gordon Jl. The effect of diet on the human gut Microbiome : a metagenomic analysis in humanized Gnotobiotic mice. Sci Transl Med. 2009;1(6):1-12.

25. den Besten G, van Eunen K, Groen AK, Venema K, Reijngoud D-J, Bakker BM. The role of short-chain fatty acids in the interplay between diet, gut microbiota, and host energy metabolism. J Lipid Res. 2013;54(9):2325-40.

26. Kuo S-M. Reviews from ASN EB 2012 symposia the interplay between fiber and the intestinal microbiome in the inflammatory response. Adv Nutr. 2013;4:16-28.

27. Wise A, Gilburt DJ. The variability of dietary fiber in laboratory-animal diets and its relevance to the control of experimental conditions. Food Cosmet Toxicol. 1980;18:643-8.

28. Cromwell GL, Cline TR, Crenshaw JD, Crenshaw TD, Easter RA, Ewan RC, et al. Variability among sources and laboratories in analyses of wheat middlings. J Anim Sci. 2000;78:2652-8.

29. Rutten AAJJL, de Groot AP. Comparison of cereal-based diet with purified diet by short-term feeding studies in rats, mice and hamsters, with emphasis on toxicity characteristics. Food Chem Toxicol. 1992;30(7):601-10.

30. Chassaing B, Miles-Brown J, Pellizzon M, Ulman E, Ricci M, Zhang L, et al. Lack of soluble fiber drives diet-induced adiposity in mice. Am J Physiol Gastrointest Liver Physiol. 2015;309(7):G528-41.

31. Dalby MJ, Ross AW, Walker AW, Correspondence PJM, Morgan PJ. Dietary uncoupling of gut microbiota and energy harvesting from obesity and glucose tolerance in mice. Cell Rep. 2017;21:1521-33.

32. Desai MS, Seekatz AM, Koropatkin NM, Kamada N, Hickey CA, Wolter M, et al. A dietary fiber-deprived gut microbiota degrades the colonic mucus barrier and enhances pathogen susceptibility. Cell. 2016;167(5): 1339-1353.e21

33. Miles JP, Zou J, Kumar M, Pellizzon M, Ulman E, Ricci M, et al. Supplementation of low- and high-fat diets with fermentable fiber exacerbates severity of DSS-induced acute colitis. Inflamm Bowel Dis. 2017;0(0):1-11.
34. Torre-Villalvazo I, Tovar AR, Ramos-Barragán VE, Cerbón-Cervantes MA Torres N. Soy protein ameliorates metabolic abnormalities in liver and adipose tissue of rats fed a high fat diet. J Nutr. 2008;138(3):462-8.

35. Adam CL, Williams PA, Garden KE, Thomson LM, Ross AW. Dose-dependent effects of a soluble dietary fibre (pectin) on food intake, adiposity, gut hypertrophy and gut satiety hormone secretion in rats. PLoS One. 2015;10(1):1-14.

36. Blay L, Michel C, Cherbut C. Prolonged intake of Fructo-oligosaccharides induces a short-term elevation of lactic acid-producing bacteria and a persistent increase in Cecal butyrate in rats. J Nutr. 1999;129:2231-5.

37. Levrat M-A, Rémésy $C$, Demigné $C$. High propionic acid fermentations and mineral accumulation in the cecum of rats adapted to different levels of inulin. J Nutr. 1991;121(11):1730-7.

38. Respondek F, Gerard P, Bossis M, Boschat L, Rabot S, Wagner A, et al. Shortchain Fructo-oligosaccharides modulate intestinal microbiota and metabolic parameters of humanized Gnotobiotic diet induced obesity mice. PLoS One. 2013;8(8):e71026.

39. Adam CL, Williams PA, Dalby MJ, Garden K, Thomson LM, Richardson AJ, et al. Different types of soluble fermentable dietary fibre decrease food intake, body weight gain and adiposity in young adult male rats. Nutr Metab (Lond). 2014;11(1):36. Available from: http://nutritionandmetabolism. biomedcentral.com/articles/10.1186/1743-7075-11-36

40. Nakamura S, Kondo N, Yamaguchi Y, Hashiguchi M, Tanabe K, Ushiroda C, et al. Daily feeding of fructooligosaccharide or glucomannan delays onset of senescence in SAMP8 mice. Gastroenterol Res Pract. 2014;2014:303184.

41. Neyrinck A, Hé EV, Piront N, De Backer F, Toussaint O, Cani P, et al. Wheat-derived arabinoxylan oligosaccharides with prebiotic effect increase satietogenic gut peptides and reduce metabolic endotoxemia in diet-induced obese mice. Nutr Diabetes. 2012;224. https://doi.org/10.1038/ nutd.2011.24

42. Collins FS, Tabak LA. Collins Tabak nature 2014 NIH calls for increased rigor and reproducibility. Nature. 2014;505:612-3.

\section{Submit your next manuscript to BioMed Central and we will help you at every step:}

- We accept pre-submission inquiries

- Our selector tool helps you to find the most relevant journal

- We provide round the clock customer support

- Convenient online submission

- Thorough peer review

- Inclusion in PubMed and all major indexing services

- Maximum visibility for your research

Submit your manuscript at www.biomedcentral.com/submit
Biomed Central 